Predicting intention and behaviour following participation in a theory-based intervention to improve gluten free diet adherence in coeliac disease

Title: Predicting intention and behaviour following participation in a theorybased intervention to improve gluten free diet adherence in coeliac disease

Authors: Dr. Kirby Sainsbury, PhD (ksai7668@uni.sydney.edu.au) $)^{1,2}$ A/Prof. Barbara Mullan, PhD (barbara.mullan@.curtin.edu.au) $)^{1,2}$ Prof. Louise Sharpe, $\mathrm{PhD}$ (louise.sharpe@ sydney.edu.au) ${ }^{2}$

1. School of Psychology and Speech Pathology, Curtin University, Perth, WA, Australia

2. School of Psychology, University of Sydney, Sydney, NSW, Australia

Corresponding author: A/Prof. Barbara Mullan, Health Psychology and Behavioural Medicine Research Group, School of Psychology and Speech Pathology, Faculty of Health Sciences, Curtin University, Perth, Australia. Phone: +61 (0)8 9266 2468; email: barbara.mullan@curtin.edu.au

Word count (excluding abstract, tables, figures, references): 3440

RCT registration details: Australian New Zealand Clinical Trials Registry; Trial ID: ACTRN12612001258842 


\title{
Predicting intention and behaviour following participation in a theory-based intervention to improve gluten free diet adherence in coeliac disease
}

\begin{abstract}
Objective: To determine whether changes in theory of planned behaviour (TPB) constructs could predict intention and gluten free diet (GFD) adherence following participation in an online theory-based intervention designed to improve adherence in coeliac disease.

Design: Theory-based process evaluation of the mechanisms of change over the course of a six-week online intervention. Measures of GFD adherence and TPB variables were administered at baseline and follow-up (immediate post-intervention: $\mathrm{n}=74$; three-month: $\mathrm{n}=68$; six-month: $\mathrm{n}=65$ ). Hierarchical regression analyses using residualised change scores were conducted at each time point (dependent variables: intention and adherence).
\end{abstract}

Results: Baseline intention and GFD adherence were the strongest predictors of follow-up intention and adherence respectively. Change in attitude accounted for significant variance in intention. Change in intention accounted for significant variance in GFD adherence immediately post-intervention; by the six-month followup change in perceived behavioural control was the stronger predictor.

Conclusions: Partial support for the hypotheses suggests that, for certain behaviours, the TPB may be relevant in explaining the mechanism of action responsible for changes in intention and behaviour following participation in a behaviour change intervention. Additional predictive pathways are also likely to exist and, in the area of GFD adherence, may include habit strength and actual behavioural control.

Keywords: theory of planned behaviour, intervention, behaviour change, coeliac disease, gluten free diet adherence 


\section{Predicting intention and behaviour following participation in a theory-based intervention to improve gluten free diet adherence in coeliac disease}

\section{Introduction}

Arguably the biggest question within the field of health psychology and behaviour change currently is whether theory-based behaviour change interventions do indeed exert their effects on behaviour via the pathways specified by the selected theory (Hardeman et al., 2002; Michie \& Abraham, 2004). In other words, whether the models commonly used in the prediction of behaviour are also able to account for behaviour change. This is an important question because even if an intervention is effective, without an understanding of the likely mechanisms of behaviour change, it is difficult to refine the intervention to develop more effective treatments (Michie, Fixsen, Grimshaw, \& Eccles, 2009; Michie, Hardeman, et al., 2008; Michie et al., 2005; Michie, Johnston, Francis, Hardeman, \& Eccles, 2008). Within the context of a theory of planned behaviour (TPB)-based intervention this would involve the demonstration that the effect of the intervention on behaviour is accounted for or mediated by changes in intention and perceived behavioural control, while the effect on intention is accounted for by changes in attitudes, subjective norm, and perceived behavioural control (Ajzen, 1991, 2011).

In a systematic review of TPB-based behaviour change interventions Hardeman et al. (2002) found only two papers that reported on the mediation of intention and/or behaviour change via TPB-relevant pathways. Both papers provided early support for the assertion that successfully targeting the social-cognitive determinants of intention and behaviour can lead to meaningful improvements in intention (Beale \& Manstead, 1991; Bowen, 1996) and behaviour (Beale \& Manstead, 1991). Since this review, the reporting of TPB-relevant mediation analyses has become more frequent. For example, in an intervention to increase walking, Darker, French, Eves, and Sniehotta (2010) found that intervention effects on intention were 


\section{Predicting intention and behaviour following participation in a theory-based intervention to improve gluten free diet adherence in coeliac disease}

mediated by changes in attitude and perceived behavioural control, while changes in perceived behavioural controlmediated the effect of the intervention on actual walking behaviour. Elliot and Armitage (2009) similarly found that changes in a particular control belief mediated the effect of their intervention on perceived behavioural control, which in turn mediated the effect on improved compliance with speed limits. Two further studies found positive results regarding the modelling of intention change via TPB pathways but failed to find support for modelling actual behaviour change (Chatzisarantis \& Hagger, 2005; Kothe, Mullan, \& Butow, 2012).

Hardeman, Kinmonth, Michie, and Sutton (2011) examined whether intention and perceived behavioural control (direct and indirect measures) measured at baseline could predict behaviour change (physical activity) over a 12-month intervention period. In contrast to the previous studies neither variable was a significant predictor of self-reported or objective behaviour change, with the total variance accounted for being very low (1-2\%). One potential explanation for why the TPB failed to predict change was temporal instability of beliefs. Indeed, prediction of behaviour is greater when the time interval between the measurement of cognitions and behaviour is reduced (McEachan, Conner, Taylor, \& Lawton, 2011), as multiple factors may influence post-measurement intentions/cognitions such that when behaviour is measured after a delay, intentions cannot be assumed to be unchanged (Ajzen, 1991).

Regardless of non-significant changes in either outcome measures or hypothesised predictors across the course of an intervention, analyses that capture change are still needed to test the mechanism of action underlying theory-based interventions, particularly when the intervention is designed to alter TPB cognitions in addition to behaviour. Unfortunately, however, the use of change scores has been associated with numerous conceptual and statistical difficulties (Cronbach \& Furby, 


\section{Predicting intention and behaviour following participation in a theory-based intervention to improve gluten free diet adherence in coeliac disease}

1970), which have made answering questions about the mechanism of action of behaviour change interventions difficult. In a recently published paper of a longitudinal study to assess the impact of TPB cognitions on car use, Armitage, Reid, and Spencer (2013) advocated for the use of residualised change scores, which overcome the difficulties associated with regular change scores. Using this method, they found that baseline intention, and changes in attitude, subjective norm, and perceived behavioural control accounted for a significant proportion of the variance in follow-up intention; and baseline behaviour and change in perceived behavioural control accounted for significant variance in follow-up behaviour.

The aim of the present paper was to conduct a theory-based process evaluation of the mechanism of action of the "Bread n' Butter... Gluten Free of Course!" intervention, using data from a previously published RCT (Sainsbury, Mullan, \& Sharpe, 2013b), and following the method suggested by Armitage et al. (2013). Briefly, coeliac disease is a chronic autoimmune disorder involving intolerance for dietary gluten, which is not only associated with short-term gastrointestinal symptoms, but is also linked to an increased risk of developing long-term health complications including cancer, osteoporosis, and infertility (Green \& Jabri, 2003). The only available treatment for coeliac disease is lifelong strict adherence to a gluten free diet (GFD), with even minor exposure being sufficient to trigger an immune response (Biagi et al., 2004; Green \& Cellier, 2007). The “Bread n’ Butter... Gluten Free of Course!" intervention was developed as a resource to improve GFD adherence in individuals with coeliac disease, and currently represents the only intervention designed for this purpose. It was based on previous qualitative and quantitative research which showed that an extended TPB model (including depression as a postintentional factor) accounted for significant variance in GFD adherence (Sainsbury \& 


\section{Predicting intention and behaviour following participation in a theory-based intervention to improve gluten free diet adherence in coeliac disease}

Mullan, 2011; Sainsbury, Mullan, \& Sharpe, 2013a), with participants who scored higher on depression measures being less likely to translate their positive intentions into good adherence (Sainsbury et al., 2013a). It included TPB-relevant behaviour change techniques to target attitudes (e.g., provide information on the healthbehaviour link), subjective norm (e.g., provide information about others' behaviour), perceived behavioural control (e.g., provide instruction), and intention (e.g., prompt intention; Abraham, Kok, Schaalma, \& Luszczynska, 2010), as well as educational material concerning coeliac disease, the need for strict adherence, and how to identify gluten free ingredients.

The intervention resulted in statistically (small-to-medium effect size) and clinically meaningful improvements in GFD adherence relative to a waitlist control group, which was increased to a medium-to-large effect amongst the participants with inadequate baseline adherence (Sainsbury et al., 2013b). No changes in TPB variables were observed within the intention-to-treat analyses; however, amongst the participants who completed the intervention as planned (per-protocol analyses) there was a significant improvement in attitudes at immediate post-intervention, and further improvement when measured again at six-month follow-up. The specific aim of this paper was to determine, in addition to the already demonstrated effectiveness of the intervention, whether such changes in TPB constructs among the sample who completed the intervention were responsible for the positive effect of the intervention on GFD adherence. As previously mentioned, this is an important question within the health psychology field as without an understanding of how an intervention worked attempts at further refinement are limited. In the context of the causal pathways posited by the TPB and which informed the design of the intervention, it was expected that changes in intention and perceived behavioural control across the course 


\section{Predicting intention and behaviour following participation in a theory-based intervention to improve gluten free diet adherence in coeliac disease}

of the intervention would predict follow-up GFD adherence; and that changes in attitude, subjective norm, and perceived behavioural control would predict follow-up intentions.

\section{Method}

\section{Measures}

The following measures were completed at baseline, immediately postintervention, and at three- and six-month follow-up. The Coeliac Dietary Adherence Test (CDAT; Leffler et al., 2009) is a seven-item questionnaire which represents the only GFD adherence measure to have been validated against the gold standard dietitian-rated estimate of adherence. The questionnaire has demonstrated test-retest reliability and face, internal, and external validity, and has been found to be superior to serological/histological analysis (the presence of antibodies and villous atrophy, which signal continued gluten exposure) in detecting inadequate GFD adherence. Scores range from 7-35, with higher scores indicating poorer adherence.

The Coeliac Disease Theory of Planned Behaviour Questionnaire (13 direct items only; Sainsbury \& Mullan, 2011) was used to assess intention, attitude, subjective norm, and perceived behavioural control in relation to maintaining a strict GFD. The full questionnaire (containing 76 indirect items and 13 direct items) was designed following a series of TPB-based elicitation interviews as per published guidelines on the construction of TPB-based questionnaires (Ajzen, 2006; Francis et al., 2004). Composite scores for the direct items administered here range from 1-7, with higher scores indicating more positive cognitions towards the GFD. The questionnaire has been shown to have good internal consistency and construct validity (Sainsbury \& Mullan, 2011).

\section{Participants and procedure}




\section{Predicting intention and behaviour following participation in a theory-based intervention to improve gluten free diet adherence in coeliac disease}

All participants were recruited through the Coeliac Society of New South Wales, Australia, and met the following inclusion criteria: biopsy-confirmed coeliac disease, GFD duration > 3 months, aged > 16 years. One hundred and eighty-nine participants completed the baseline questionnaires and were randomised to the intervention $(n=101)$ or waitlist control group $(n=88$; Sainsbury et al., 2013b). At baseline, the mean GFD adherence score was $12.20(S D=3.44$; range $=7-28)$; $58.9 \%$ of the sample had excellent or very good adherence, $33.2 \%$ moderate, and 7.9\% fair-to-poor adherence.

Fifty intervention group participants (49.5\%) completed the intervention and post-intervention survey and a further 24 waitlist control group participants subsequently completed the program following the initial waitlist period. Thus, 74 participants ( $36.5 \%$ of whom had inadequate GFD adherence at baseline) were included in the immediate post-intervention analyses. The majority of this sample also completed the follow-up questionnaires (three-month follow-up: $n=68$; six-month follow-up: $n=65$ ). Although there was a high level of attrition across the course of the intervention, there were no differences between completers and those lost to follow-up on GFD adherence or any of the measured baseline variables $(p>.05)$. All questionnaires and the six intervention modules were completed online. The study was conducted according to the protocol approved by the University Human Research Ethics Committee and all participants provided informed consent prior to completion of the baseline questionnaires. For more details on the sample and intervention content and procedure please refer to related papers (Sainsbury et al., 2013b; Sainsbury, Mullan, \& Sharpe, 2013c).

\section{Data analysis}




\section{Predicting intention and behaviour following participation in a theory-based intervention to improve gluten free diet adherence in coeliac disease}

The analyses used to assess the impact of change in TPB cognitions on follow-up intention and GFD adherence were based on those reported by Armitage et al. (2013) in their paper assessing causal relationships within the TPB. Specifically, based on the criticisms of change scores (Cronbach \& Furby, 1970), they computed residualised change scores. In contrast to standard change scores, residualised change scores serve to partial out the part of follow-up data that is linearly predictable from baseline, therefore reducing the correlation between baseline scores and change across the course of an intervention, while still allowing for the important impact of past behaviour to be captured (Armitage et al., 2013). Past behaviour has frequently been included as an extension to the TPB, and was included here based on the arguments made by Armitage et al. (2013) - that is, controlling for past behaviour provides a test of the sufficiency of the TPB and ensures that any additional variance accounted for is explained by changes across time. After controlling for baseline scores, a significant residualised change score in step 2 can therefore be interpreted as indicating that change in that construct was at least partially responsible for the effect of the intervention on the outcome of interest.

A hierarchical regression analysis was used to determine the impact of changes (residualised) in attitude, subjective norm, and perceived behavioural control on intentions measured at immediate post-intervention, three-month, and six-month follow-up, controlling for baseline intention at step 1. Similarly, a regression analysis was conducted to determine the impact of changes (residualised) in intention and perceived behavioural control on GFD adherence measured at each of the three follow-up time points, again controlling for baseline adherence at step 1. Analyses were conducted on the sample of participants who completed the intervention and each of the three follow-up questionnaires (immediate-post intervention: $n=74$; 


\section{Predicting intention and behaviour following participation in a theory-based intervention to improve gluten free diet adherence in coeliac disease}

three-month follow-up: $n=68$; six-month follow-up: $n=65$ ). Finally, mediation analyses (bootstrapping procedure: Preacher and Hayes, 2008) were used to determine whether the effect of attitude change on GFD adherence (immediate postintervention) was mediated by intention change. This analysis was based on 5000 resamples.

\section{Results ${ }^{1}$}

\section{Predicting follow-up intention}

At step 1, baseline intention accounted for $36.2 \%$ of the variance in immediate post-intervention intention. The theory of planned behaviour pre-intention variables (residualised change scores) accounted for a further $14 \%$, although only attitude change, not subjective norm change or perceived behavioural control change, made a significant independent contribution (see Table 1).

The same analysis was repeated with (1) three-month follow-up intention, and (2) six-month follow-up intention as the dependent variable, and both revealed that changes (residualised) in attitude, subjective norm, and perceived behavioural control did not contribute to the prediction of intention over and above the influence of baseline intention (Step 1: $R^{2}=.249$ and .193 respectively, both $p<.001$; step 2: $p>$ $.05)$

\section{INSERT TABLE 1 HERE}

\section{Predicting follow-up GFD adherence}

\footnotetext{
${ }^{1}$ Based on the results of the Hardeman et al. (2011) study whereby it was suggested that the demonstration of the predictive capacity of the TPB cross-sectionally may be seen as a pre-requisite for successfully modelling intention and behaviour change, cross-sectional linear regression analyses were conducted to confirm the fit of the TPB in the baseline and post-intervention samples. Baseline $(\mathrm{N}=189)$ : Attitude $(\beta=.138, p<.05)$, subjective norm $(\beta=.040, p>.05)$, and $\mathrm{PBC}(\beta=.573, p<.001)$ accounted for $43.7 \%$ of the variance in intention $\left(R^{2}=.437, F_{3,185}=47.94, p<.001\right)$. Intention $(\beta=-.226, p<.01)$ and $\operatorname{PBC}(\beta=$ $-.412, p<.001)$ accounted for $34.2 \%$ of the variance in GFD adherence $\left(R^{2}=.342, F_{2,186}=48.28, p<.001\right)$. Post-intervention $(\mathrm{N}=134$; intervention: $\mathrm{n}=70$; waitlist: $\mathrm{n}=64)$ : Attitude $(\beta=.367, p<.05)$, subjective norm $(\beta=.033, p>.05)$, and $\mathrm{PBC}(\beta=$ $.396, p<.001)$ accounted for $46.8 \%$ of the variance in intention $\left(R^{2}=.468, F_{3,130}=38.16, p<.001\right)$. Intention $(\beta=-.242, p<$ $.01)$ and $\operatorname{PBC}(\beta=-.454, p<.001)$ accounted for $39.9 \%$ of the variance in GFD adherence $\left(R^{2}=.399, F_{2,131}=43.44, p<.001\right)$.
} 


\section{Predicting intention and behaviour following participation in a theory-based intervention to improve gluten free diet adherence in coeliac disease}

At step 1, baseline GFD adherence accounted for 55.5\% of the variance in immediate post-intervention GFD adherence (see Table 2). Residualised change scores for intention and perceived behavioural control accounted for a further $6.1 \%$ at step 2, although only intention change, not perceived behavioural control change, made a significant independent contribution.

The same analysis using three-month follow-up GFD adherence as the dependent variable revealed that baseline intention again accounted for a significant amount of the variance $(22.1 \%)$; changes in intention and perceived behavioural control (residualised change scores) added a further $4.5 \%$ to the prediction but were not significant.

Finally, the analysis using six-month follow-up GFD adherence as the dependent variable showed that baseline GFD adherence accounted for $31.6 \%$ of the variance, while changes in intention and perceived behavioural control (residualised change scores) added $9 \%$ to the model at step 2 . In contrast to the first analysis, change in perceived behavioural control, but not change in intention, made a significant independent contribution.

The bootstrapping procedure showed that the change in intention was not a significant mediator of the relationship between changes in attitude and GFD adherence measured at immediate post-intervention $(95 \% \mathrm{CI}=-2.08-0.7)$.

\section{INSERT TABLE 2 HERE}

\section{Discussion}

The aim of this study was to determine whether changes in TPB variables resulting from participation in an online theory-based intervention could explain intention and GFD adherence, as measured at three follow-up time points. While the TPB has previously been shown to provide a good fit to adherence data in coeliac 


\section{Predicting intention and behaviour following participation in a theory-based intervention to improve gluten free diet adherence in coeliac disease}

disease (Sainsbury \& Mullan, 2011; Sainsbury et al., 2013a), mixed support for the present change-related hypotheses was found. That is, changes in attitude accounted for significant variance in follow-up intention, and changes in intention accounted for variance in follow-up adherence; however, changes in the TPB variables of subjective norm and perceived behavioural control were not related to subsequent intention or adherence, and nor was the mediation analysis significant. In both cases, the strongest predictor of follow-up scores was the corresponding baseline variable, confirming the important role of past behaviour in informing future behaviour, as well as demonstrating the test-retest reliability of the intention and behaviour measures. Methodologically, the use of residualised change scores (which partial out the already accounted for influence of baseline scores and the correlation between baseline and change) in the second step of each respective analysis adds weight to the conclusion that changes in TPB constructs (attitude and intention) across the course of the intervention were partially responsible for the positive effect of the intervention of intention and behaviour.

The effectiveness of targeting attitudes as a means to changing intention, as evidenced here, is consistent with previous findings (Beale \& Manstead, 1991; BoothButterfield \& Reger, 2004; Bowen, 1996; Chatzisarantis \& Hagger, 2005; Darker et al., 2010; Kothe et al., 2012). It was, however, interesting that change in attitude was only significant in the immediate post-intervention analysis and not the three- or sixmonth follow-up analyses. This is despite the observation that attitude scores had improved further at six-month follow-up (compared to baseline, immediate postintervention, and three-month follow-up) amongst the sample who completed the intervention - likely reflecting the positive feedback loop between behaviour change (improved adherence) and subsequently improved cognitions. Thus, it appears that 


\section{Predicting intention and behaviour following participation in a theory-based intervention to improve gluten free diet adherence in coeliac disease}

while changes in attitude are important in the initiation of positive intentions, the longer that an individual with coeliac disease remains on the GFD, the less influence attitudes have in maintaining their positive intentions. Rather, it may be the case that factors such as habit (discussed later) become more important.

In contrast, changes in subjective norm and perceived behavioural control were not significant predictors of intention at any time point. The lack of predictive power of subjective norm is not surprising as this variable did not appear to be an important consideration in informing adherence decisions when assessed in an interview study, and nor was subjective norm significant in the prediction of intention to strictly adhere to a GFD in several cross-sectional studies (Sainsbury \& Mullan, 2011; Sainsbury et al., 2013a). Regarding perceived behavioural control, high baseline scores and non-significant change across the course of the intervention were the likely reasons for its failure to account for variance in intention.

Comparison of the pattern of significant predictors of GFD adherence across the three time points also revealed some interesting findings. Specifically, while intention change was significant in the prediction of immediate post-intervention GFD adherence, by six-month follow-up, change in perceived behavioural control was the more important (and only significant TPB) predictor; neither intention nor perceived behavioural control change were significant in three-month follow-up adherence scores. According to the TPB, perceived behavioural control should be a stronger predictor of behaviour in situations where the translation of intention into behaviour is likely to be hindered by relevant external factors (Ajzen, 1991). Given the difficulty of achieving and maintaining strict GFD adherence, it is possible that perceived behavioural control, as measured here, failed to adequately tap actual behavioural control. Therefore, although the intervention positively impacted the 


\section{Predicting intention and behaviour following participation in a theory-based intervention to improve gluten free diet adherence in coeliac disease}

management of the GFD (as evidenced by improved adherence scores), this was not reflected in the perceived behavioural control measurement and therefore meant that change in perceived behavioural control did not initially contribute to the change in adherence. Further, this pattern strongly suggests that while intention is initially an important determinant of good adherence, in order to maintain this state it is necessary for individuals to develop feelings of control over their ability to correctly identify gluten-free foods, avoid contamination, and develop confidence in their capacity to overcome external barriers to adherence.

The pattern of significant predictors is also consistent with predictions derived from temporal self-regulation theory (Hall \& Fong, 2007), which differentiates between discrete or one-off behaviours and repetitive behaviours, such as GFD adherence. Specifically, the model states that under supportive environmental conditions, behavioural prepotency (similar to past behaviour, which was the most significant predictor of subsequent behaviour here) and self-regulatory capacity are likely to be the best predictors of behaviour, with intentions only being secondarily important. In contrast, when the environment is unsupportive of behaviour, intentions and self-regulation should both be directly predictive of behaviour, with the influence of past behaviour being lessened (Hall \& Fong, 2007).

Another aspect of behavioural prepotency that has been used to operationalise this construct in many studies is habit strength - the extent to which a particular behaviour has become automatic (Hall \& Fong, 2007). Similarly, when behaviour has become automatised it is likely to require less conscious motivation to enact and instead rely more heavily on positive perceptions of one's ability to overcome barriers to adherence. Indeed, a recent study found that a three-way interaction between habit strength, perceived behavioural control, and intention had a significant moderating 


\section{Predicting intention and behaviour following participation in a theory-based intervention to improve gluten free diet adherence in coeliac disease}

effect on the intention-GFD adherence relationship, such that intentions were only predictive of GFD adherence when perceived behavioural control was low and habits were high (Kothe, Sainsbury, Smith, \& Mullan, 2014). Consistent with the six-month follow-up GFD adherence results here, this finding suggests that positive intentions in the absence of the parallel development of confidence and strong habits is not enough to prompt adequate adherence.

\section{Limitations and conclusions}

This study had several limitations that should be considered when interpreting the results. Firstly, there was a high level of attrition from the intervention, which meant that the analyses of change could only be conducted on the reduced sample of participants rather than the whole study cohort. Second and related, individuals with varying levels of adherence were recruited to the intervention and, as such, approximately half the participants were already exhibiting excellent or very good adherence prior to participation (Sainsbury et al., 2013b). When combined with substantial attrition, this meant that large changes across the course of the intervention were unlikely. Restricted variance in the outcome measures may therefore have limited the ability of the theory to account for a greater proportion of follow-up intention and behaviour. Replication in a larger sample, including a greater proportion of individuals who are exhibiting inadequate adherence, would strengthen the conclusions. Finally, the recruitment of participants from the Coeliac Society may have led to a bias whereby those who were more invested in their health were more likely to enrol and stay in the trial. As mentioned, there were no differences in baseline variables between completers and those who dropped out; however, the representativeness of the sample amongst the greater population of people suffering from coeliac disease cannot be guaranteed. 


\section{Predicting intention and behaviour following participation in a theory-based intervention to improve gluten free diet adherence in coeliac disease}

The failure of the TPB to improve behaviour in several recent interventions (Hardeman et al., 2011; Kothe et al., 2012), has led to the tentative view that while the TPB represents a sound theory for the prediction of behaviour, its ability to account for change in behaviour may be more limited. Despite the above-mentioned limitations, the present results challenge this view and suggest that, for particular behaviours, the TPB (when extended to include past behaviour) can provide useful insight into the mechanisms underlying intention and behaviour change. Having said this, the unaccounted for portion of the variance in both intention and behaviour also suggests that further research is needed to determine additional pathways via which improvements in GFD adherence can be achieved. In particular, it appears that the inclusion of measures of habit strength and actual behaviour control may be useful in this endeavour, as both this and recent research (Kothe, Sainsbury, Smith, \& Mullan, in press) suggests that improvements in intention alone are not likely to lead to sustained behaviour change. In addition to the significant clinical implications for the management of adherence in coeliac disease (Sainsbury et al., 2013b), this study therefore adds to the growing body of literature concerning the theoretical mechanisms underlying successful behaviour change interventions (e.g., Darker et al., 2010; Elliot \& Armitage, 2009; Hardeman et al., 2002; Hardeman et al., 2011; Kothe et al., 2012).

\section{Acknowledgements}


Predicting intention and behaviour following participation in a theory-based intervention to improve gluten free diet adherence in coeliac disease

The authors would like to thank the staff of the Coeliac Society of NSW, Australia for assistance with database screening and recruitment; and the members of the Coeliac Society who participated in the intervention. LS is currently being supported by a Senior NHMRC Research Fellowship. 
Predicting intention and behaviour following participation in a theory-based intervention to improve gluten free diet adherence in coeliac disease

Table 1. Hierarchical regression analysis predicting immediate post-intervention intention scores

\begin{tabular}{lllll}
\hline Variable & $B$ & $\beta$ & $R^{2}(\Delta)$ & $F$ \\
\hline Step 1 & & & & \\
$\quad$ Baseline intention & .672 & $.602 * * *$ & .362 & $40.91^{* * *}$ \\
Step 2 & & & & \\
Baseline intention & .596 & $.533^{* * *}$ & & \\
Attitude $^{\mathrm{a}}$ & .454 & $.363^{* * *}$ & & \\
Subjective norm $^{\mathrm{a}}$ & .013 & .013 & & \\
PBC $^{\mathrm{a}}$ & .077 & .057 & $.503(.14)$ & $17.44^{* * *}$ \\
\hline
\end{tabular}

Note: ${ }^{\mathrm{a}} \mathrm{TPB}$ variables used in analyses are residualised change scores (from baseline

to immediate post-intervention); $\mathrm{PBC}=$ perceived behavioral control; $* * * p<.001$ 


\section{Predicting intention and behaviour following participation in a theory-based intervention to improve gluten free diet adherence in coeliac disease}

Table 2. Hierarchical regression analyses predicting GFD adherence scores at three time points

\begin{tabular}{lccc}
\hline Variable & $B$ & $R^{2}(\Delta)$ & $F$ \\
\hline Immediate post-intervention GFD adherence &
\end{tabular}

\section{Step 1}

Baseline adherence

.751

$.745^{* * *}$

.555

$89.64 * * *$

Step 2

$\begin{array}{lllll}\text { Baseline adherence } & .726 & .720 * * * & & \\ \text { Intention }^{\mathrm{a}} & -1.155 & -.230 * * & & \\ \text { PBC }^{\mathrm{a}} & -.536 & -.095 & .616(.061) & 37.42 * * *\end{array}$

Three-month follow-up GFD adherence

Step 1

$\begin{array}{lllll}\text { Baseline adherence } & .389 & .470 * * * & .221 & 18.69 * * *\end{array}$

Step 2

$\begin{array}{lll}\text { Baseline adherence } & .441 & .532 * * * \\ \text { Intention }^{\mathrm{b}} & -.399 & -.085 \\ \text { PBC }^{\mathrm{b}} & -.706 & -.177\end{array}$

$.265(.045) \quad 7.70 * * *$

Six-month follow-up GFD adherence

Step 1

Baseline adherence

.451

$.565^{* * *}$

.319

$29.58 * * *$

Step 2

$\begin{array}{lllll}\text { Baseline adherence } & .528 & .662 * * * & & \\ \text { Intention }^{\mathrm{c}} & -.288 & -.064 & & \\ \text { PBC }^{\mathrm{c}} & -1.107 & -.287 * * & .410(.090) & 14.10^{* * *}\end{array}$

Note: GFD = gluten free diet; $\mathrm{PBC}=$ perceived behavioural control; TPB variables used in analyses are residualised change scores ( ${ }^{\mathrm{a}}$ change from baseline to immediate post intervention; ${ }^{\mathrm{b}}$ change from baseline to three-month follow-up; ${ }^{\mathrm{c}}$ change from baseline to six-month follow-up; adherence measure: higher scores indicate poorer adherence; $* * * p<.001, * * p<.01$ 


\section{Predicting intention and behaviour following participation in a theory-based intervention to improve gluten free diet adherence in coeliac disease}

References

Abraham, C., Kok, G., Schaalma, H., \& Luszczynska, A. (2010). Health promotion. In P. R. Martin, F. Cheung, M. Kyrios, L. Littlefield, L. Knowles, M. Overmier \& J. M. Prieto (Eds.), The international association of applied psychology handbook of applied psychology. Oxford: Wiley-Blackwell.

Ajzen, I. (1991). The theory of planned behavior. Organisational Behaviour and Human Decision Processes, 50, 179-211.

Ajzen, I. (2006). Constructing a TPB questionnaire: Conceptual and methodological considerations. Retrieved 18th September 2009, from http://people.umass.edu/aizen/pdf/tpb.measurement.pdf

Ajzen, I. (2011, 16/11/11). Behavioural interventions based on the Theory of Planned Behaviour. Retrieved 28th March 2012, from http://people.umass.edu/aizen/pdf/tpb.intervention.pdf

Armitage, C. J., Reid, J. C., \& Spencer, C. P. (2013). Changes in cognition and behaviour: A causal analysis of single-occupancy car use in a rural community. Transportmetrica A: Transport Science, 9, 1-10.

Beale, D. A., \& Manstead, A. S. R. (1991). Predicting mothers' intentions to limit frequency of infants' sugar intake: Testing the theory of planned behaviour. Journal of Applied Social Psychology, 21, 409-431.

Biagi, F., Campanella, J., Martucci, S., Pezzimenti, D., Ciclitira, P. J., Ellis, H. J., \& Corazza, G. R. (2004). A milligram of gluten a day keeps the mucosal recovery away: A case report. Nutrition Reviews, 62, 360 -363.

Booth-Butterfield, S., \& Reger, B. (2004). The message changes belief and the rest is theory: The "1\% or less" milk campaign and reasoned action. Preventative Medicine, 39, 581-588.

Bowen, A. M. (1996). Predicting increased condom use with main partners: Potential approaches to intervention. Drugs and Society, 9, 57-74.

Chatzisarantis, N. L. D., \& Hagger, M. S. (2005). Effects of a brief intervention based on the theory of planned behaviour on leisure-time physical activity participation. Journal of Sport and Exercise Psychology, 27, 470-487.

Cronbach, L. J., \& Furby, L. (1970). How should we measure "change": Or should we? Psychological Bulletin, 74, 68-80.

Darker, C., French, D., Eves, F., \& Sniehotta, F. (2010). An intervention to promote walking amongst the general population based on an 'extended' theory of planned behaviour: A waiting list randomised controlled trial. Psychology and Health, 25, 71-88.

Elliot, M. A., \& Armitage, C. J. (2009). Promoting drivers' compliance with speed limits: Testing an intervention based on the theory of planned behaviour. British Journal of Psychology, 100, 111-132.

Francis, J. J., Eccles, M. P., Johnston, M., Walker, A., Grimshaw, J., Foy, R., . . . Bonetti, D. (2004). Constructing questionnaires based on the theory of planned behaviour: A manual for health services researchers. Newcastle upon Tyne: Centre for Health Services Research.

Green, P. H. R., \& Cellier, C. (2007). Celiac disease. The New England Journal of Medicine, 357, 1731-1743.

Green, P. H. R., \& Jabri, B. (2003). Coeliac disease. Lancet, 362, 383-391. 


\section{Predicting intention and behaviour following participation in a theory-based intervention to improve gluten free diet adherence in coeliac disease}

Hall, P. A., \& Fong, G. T. (2007). Temporal self-regulation theory: A model for individual health behaviour. Health Psychology Review, 1, 6-52.

Hardeman, W., Johnston, M., Johnston, D., Bonetti, D., Wareham, N., \& Kinmonth, A. L. (2002). Application of the theory of planned behaviour in behaviour change interventions: A systematic review. Psychology and Health, 17, 123158.

Hardeman, W., Kinmonth, A. L., Michie, S., \& Sutton, S. (2011). Theory of planned behaviour cognitions do not predict self-reported or objective physical activity levels or change in the ProActive trial. British Journal of Health Psychology, 16, 135-150.

Kothe, E., Mullan, B., \& Butow, P. (2012). Promoting fruit and vegetable consumption. Testing an intervention based on the theory of planned behaviour. Appetite, 58, 997-1004.

Kothe, E., Sainsbury, K., Smith, L., \& Mullan, B. (in press). Explaining the intentionbehaviour gap in gluten free diet adherence: The moderating roles of habit and perceived behavioural control. Journal of Health Psychology.

Leffler, D. A., Dennis, M., Edwards-George, J., Jamma, S., Magge, S., Cook, E. F., . . . Kelly, C. P. (2009). A simple validated gluten-free diet adherence survey for adults with celiac disease. Clinical Gastroenterology and Hepatology, 7, 530536.

McEachan, R. R. C., Conner, M., Taylor, N. J., \& Lawton, R. J. (2011). Prospective prediction of health-related behaviours with the theory of planned behaviour: A meta-analysis. Health Psychology Review, 5, 97-144.

Michie, S., \& Abraham, C. (2004). Interventions to change health behaviours: Evidence-based or evidence-inspired? Psychology and Health, 19, 29-49.

Michie, S., Fixsen, D., Grimshaw, J., \& Eccles, M. (2009). Specifying and reporting complex behaviour change interventions: The need for a scientific method. Implementation Science, 4.

Michie, S., Hardeman, W., Fanshawe, T., Prevost, A. T., Taylor, L., \& Kinmonth, A. L. (2008). Investigating theoretical explanations for behaviour change: The case study of ProActive. Psychology and Health, 23, 25-39.

Michie, S., Johnston, M., Abraham, C., Lawton, R., Parker, D., \& Walker, A. (2005). Making psychological theory useful for implementing evidence based practice: A consensus approach. Quality and Safety in Health Care, 14, 26-33.

Michie, S., Johnston, M., Francis, J. J., Hardeman, W., \& Eccles, M. (2008). From theory to intervention: Mapping theoretically derived behavioural determinants to behaviour change techniques. Applied Psychology, 57, 660680 .

Sainsbury, K., \& Mullan, B. (2011). Measuring beliefs about gluten free diet adherence in adult coeliac disease using the theory of planned behaviour. Appetite, 56, 476-483.

Sainsbury, K., Mullan, B., \& Sharpe, L. (2013a). Gluten free diet adherence in coeliac disease: The role of psychological symptoms in bridging the intentionbehaviour gap Appetite, 61, 52-58.

Sainsbury, K., Mullan, B., \& Sharpe, L. (2013b). A randomized controlled trial of an online intervention to improve gluten free diet adherence in celiac disease.

American Journal of Gastroenterology, 108, 811-817. doi:

10.1038/ajg.2013.47 
Predicting intention and behaviour following participation in a theory-based intervention to improve gluten free diet adherence in coeliac disease

Sainsbury, K., Mullan, B., \& Sharpe, L. (2013c). Reduced quality of life in coeliac disease is more strongly associated with depression than gastrointestinal symptoms. Journal of Psychosomatic Research, 75, 135-141. 